\title{
Trapping of Cold Excitons in Quantum Well Structures with Laser Light
}

\author{
A. T. Hammack, ${ }^{1}$ M. Griswold, ${ }^{1}$ L. V. Butov, ${ }^{1}$ L. E. Smallwood, ${ }^{2}$ A. L. Ivanov, ${ }^{2}$ and A. C. Gossard ${ }^{3}$ \\ ${ }^{1}$ Department of Physics, University of California at San Diego, La Jolla, California 92093-0319, USA \\ ${ }^{2}$ Department of Physics and Astronomy, Cardiff University, Cardiff CF24 3YB, United Kingdom \\ ${ }^{3}$ Materials Department, University of California at Santa Barbara, Santa Barbara, California 93106-5050, USA
}

(Received 8 March 2006; published 8 June 2006)

\begin{abstract}
Optical trapping and manipulation of neutral particles has led to a variety of experiments from stretching DNA-molecules to trapping and cooling of neutral atoms. An exciting recent outgrowth of the technique is an experimental implementation of atom Bose-Einstein condensation. In this Letter, we propose and demonstrate laser-induced trapping for a new system-a gas of excitons in quantum well structures. We report on the trapping of a highly degenerate Bose gas of excitons in laser-induced traps.
\end{abstract}

Lasers enable a precise and noninvasive application of force while also providing high speed control of the trapping field. This allows in situ trapping and control for a rich variety of small neutral particles. Since their origin three decades ago, laser based traps have been key devices in the advancement of atomic physics and biophysics; for reviews see Refs. [1-4].

In biology, the applications of optical dipole traps, also known as optical tweezers, enable direct in vivo manipulation of viruses, cells, and even individual organelles within the cells. The use of optical tweezers has also enabled probing of the mechanical properties of DNA and the forces applied by various molecular motors found in cells $[4,5]$.

In atomic physics, the use of the Doppler cooling technique [6] by sets of counter propagating lasers is employed to form an "optical molasses" containing atoms viscously confined at microkelvin temperatures. The introduction of optical tweezers to this molasses enabled the first 3D stable trap for atoms by capturing them from the surrounding molasses. Following this initial trapping of atoms, much work was devoted to the creation of larger volume magneto-optical traps (MOTs) to enhance the achievable densities of trapped atoms [1-4]. It was specifically these MOTs that led to the first realizations of Bose-Einstein condensation (BEC) in atoms [7-9]. Since this initial realization of BEC, interest has returned again to optical traps, which can be used to study magnetic effects on BEC, such as Feshbach resonances, without the complexity added by disrupting the magnetic field used in MOTs [14]. The possibility of patterning and controlling the potential profile by laser excitation is also effectively employed in studies of atom BEC in optical lattices [10].

In this Letter, we propose and demonstrate laser-induced trapping for a new system-a gas of excitons in coupled quantum wells (CQWs). Since the quantum degeneracy temperature scales inversely with the mass, quantum exciton gases can be achieved at temperatures of about $1 \mathrm{~K}$ [11], several orders of magnitude higher than quantum atom gases $[12,13]$. Indeed, the transition from a classi- cal to quantum gas occurs when bosons are cooled to the point where the thermal de Broglie wavelength $\lambda_{\mathrm{dB}}=$ $\sqrt{2 \pi \hbar^{2} /\left(m k_{\mathrm{B}} T\right)}$ is comparable to the interparticle separation (for instance, BEC takes place when $n \lambda_{\mathrm{dB}}^{3}=2.612$ in 3D systems) and the transition temperature for excitons in $\mathrm{GaAs} /(\mathrm{Al}, \mathrm{Ga}) \mathrm{As}$ QWs reaches a value of $T_{\mathrm{dB}}=$ $2 \pi \hbar^{2} n_{2 \mathrm{D}} /\left(m g k_{\mathrm{B}}\right) \approx 3 \mathrm{~K}$ for the exciton density per spin state $n_{2 \mathrm{D}} / g=10^{10} \mathrm{~cm}^{-2}$ (the exciton spin degeneracy $g=4$ and the exciton mass $m=0.22 m_{0}$ for GaAs/ (Al, Ga)As QWs [14], where $m_{0}$ is the free electron mass). Because of their long lifetime and high cooling rate, indirect excitons in a CQW [Fig. 1(a)] form a system where a cold and dense exciton gas can be created, with temperatures well below $1 \mathrm{~K}$ and densities above $10^{10} \mathrm{~cm}^{-2}$ [14]. Therefore, we chose indirect excitons for development of a method to trap cold excitons with

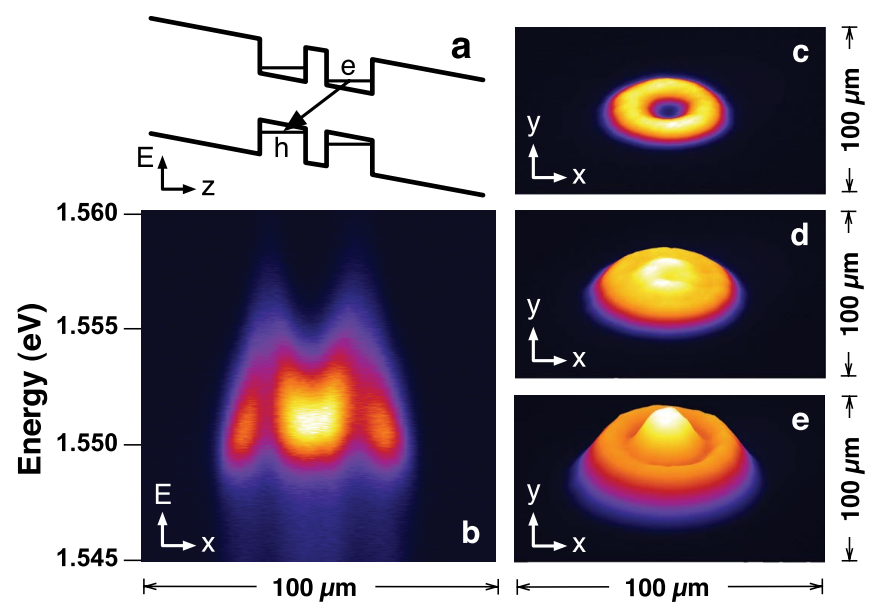

FIG. 1 (color online). Images of laser-induced trapping of excitons. (a) Energy band diagram of the CQW structure; $e$ is electron; $h$, hole. (b) Image of the PL signal in $E$ - $x$ coordinates. (c), (d), (e) Experimental $x-y$ plots of the PL intensity from indirect excitons created by $532 \mathrm{~nm} \mathrm{cw}$ laser excitation in a $30 \mu \mathrm{m}$ diameter ring on the CQW sample. For (c), (d), (e) the excitation powers are $P_{\mathrm{ex}}=10,35,100 \mu \mathrm{W}$ and for (b) $P_{\mathrm{ex}}=75 \mu \mathrm{W}$. Sample temperature $T_{\mathrm{b}}=1.4 \mathrm{~K}$. 
laser light. This technique opens a pathway towards high speed control of quantum gases of bosons in semiconductors-quantum exciton gases.

The possibility of exciton confinement and manipulation in potential traps attracted considerable interest in earlier studies. Pioneered by the electron-hole liquid confinement in the strain-induced traps [15], exciton confinement has been implemented in various traps: strain-induced traps $[16,17]$, traps created by laser-induced local interdiffusion [18], magnetic traps [19], and electrostatic traps [20-22].

The principle underlying the new method of laserinduced exciton trapping is described below. The CQW geometry [14] is engineered so that the interaction between excitons is repulsive: Indirect excitons, formed from electrons and holes that are confined to different QWs by a potential barrier, behave as dipoles oriented perpendicular to the plane, and an increasing exciton density causes an increase of the interaction energy [23-25]. The repulsive character of the interaction is evidenced in experiment as a positive and monotonic line shift with increasing density [14]. Because of the repulsive interaction, a ring-shaped laser spot should form a potential trap with the energy minimum at the ring center. Similarly to all optical traps, an important advantage of the laser-induced exciton trapping is the possibility of controlling the trap in situ by varying the laser intensity in space and time. Moreover, the excitons at the trap center are cold since they are far from the hot laser excitation ring. The long lifetimes of the indirect excitons allow them to travel to the trap center, due to their drift and diffusion, before optical recombination. This leads to accumulation of a cold and dense exciton gas at the trap center. The implementation of this idea is described below.

In our experiments, the spatial $x-y$ photoluminescence (PL) pattern is acquired by a nitrogen-cooled CCD camera after spectral selection by an interference filter chosen to match the indirect exciton energy exclusively. As a result, we are able to remove the low-energy bulk emission that otherwise dominates the spectrum under the laser excitation area. This allows the direct visualization of the indirect exciton PL emission intensity profile in spatial coordinates [see Figs. 1(c)-1(e)]. In addition, in Fig. 1(b) we plot the exciton PL in the energy-coordinate plane as measured when a slit along the diameter of the ring is dispersed by a spectrometer without spectral selection by an interference filter. Our investigations determined that a laser excitation ring with a diameter of $30 \mu \mathrm{m}$ and a ring thickness following a Gaussian profile of FWHM $=2 \sigma \simeq 7 \mu \mathrm{m}$ provided the optimal conditions for our CQW sample. The ring-shaped $\mathrm{cw}$ laser excitation was performed by a $\mathrm{Nd}: \mathrm{YVO}_{4}$ laser at $532 \mathrm{~nm}$, or a $\mathrm{HeNe}$ laser at $633 \mathrm{~nm}$, or a Ti:Sapphire laser tuned to the direct exciton resonance of $788 \mathrm{~nm}$. Spatial and spectral features were essentially similar for all excitation wavelengths investigated. In the experiments with excitation above the $(\mathrm{Al}, \mathrm{Ga}) \mathrm{As}$ barrier $(\lambda=532$ or $633 \mathrm{~nm})$, photoexcited unbalanced charges and the external ring are created, while in the experiments at nearly resonant excitation $(\lambda=788 \mathrm{~nm})$, no photoexcited unbalanced charges or external ring are created [14]. Comparison of these two experiments has shown that the charge imbalance and external ring make no noticeable effect on the exciton trapping. All experimental data presented here are from a set of $532 \mathrm{~nm}$ excitation ring data taken with excitation powers $P_{\mathrm{ex}}$ in the range $1-1000 \mu \mathrm{W}$ and with gate voltage $V_{\mathrm{g}}=1.4 \mathrm{~V}$. The CQW structure investigated is grown by molecular beam epitaxy and contains two $8 \mathrm{~nm}$ GaAs QWs separated by a $4 \mathrm{~nm}$ $\mathrm{Al}_{0.33} \mathrm{Ga}_{0.67}$ As barrier (details on the CQW structures can be found in Ref. [14]). The indirect excitons in the CQW structure are formed from electrons and holes confined to different QWs [Fig. 1(a)]. Because of the separation between the electron and hole layers in the CQW structure, the intrinsic radiative lifetimes of the optically active indirect excitons exceed that of regular direct excitons by orders of magnitudes, and are $42 \mathrm{~ns}$ at $V_{\mathrm{g}}=1.4 \mathrm{~V}$ [14].

As can be seen in Figs. 1(c)-1(e) and 2(a), for low excitation powers the PL profile follows the laser excitation ring; however, with increasing excitation power a spatial PL peak emerges at the center of the laser excitation ring, indicating the accumulation of a cold and dense exciton gas. The exciton degeneracy at the trap center $N_{E=0}=\exp \left(T_{\mathrm{dB}} / T\right)-1$ [26] can be estimated from the exciton density and temperature. The exciton density $n_{2 \mathrm{D}}=\varepsilon_{\mathrm{b}} \delta E /\left(4 \pi e^{2} d\right)$ is measured directly by the exciton energy shift $\delta E$, where $d=12 \mathrm{~nm}$ is the separation between the electron and hole layers for our samples and $\varepsilon_{\mathrm{b}}$ is

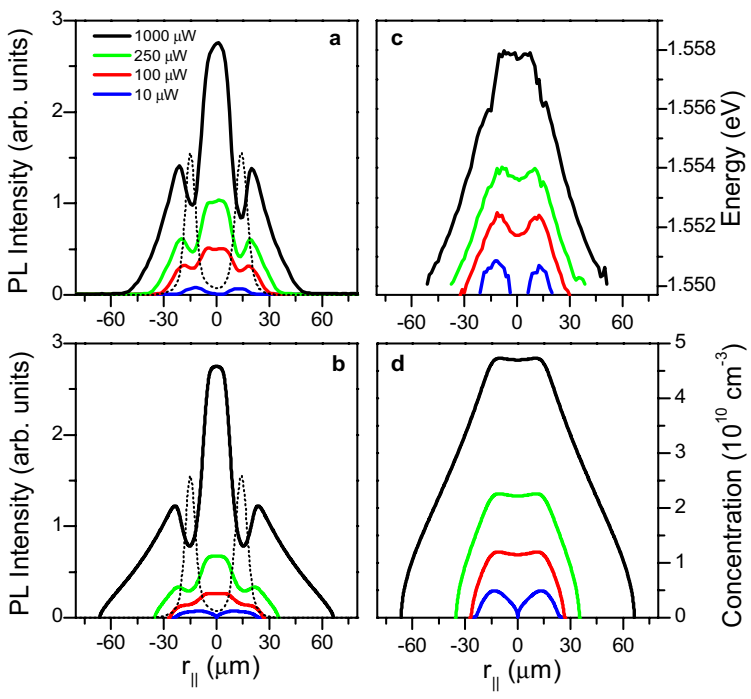

FIG. 2 (color online). Spatial profiles of the PL intensity and energy for the excitons in the laser-induced trap. (a) Measured PL intensity, (b) calculated PL intensity, (c) measured energy position of the PL line, and (d) calculated exciton concentration against radius $r_{\|}$for four optical excitation powers $P_{\mathrm{ex}}$. The vertical axes of (c) and (d) cover the same range due to the relation $\delta E=4 \pi e^{2} n_{2 \mathrm{D}} d / \varepsilon_{\mathrm{b}}$, where $d=12 \mathrm{~nm}$ for our sample. The ring-shaped profile of the laser excitation is shown by the thin dotted lines in (a) and (b). Sample temperature $T_{\mathrm{b}}=1.4 \mathrm{~K}$. 
the background dielectric constant [23-25] $\left(n_{2 \mathrm{D}}=\right.$ $10^{10} \mathrm{~cm}^{-2}$ for $\delta E \simeq 1.6 \mathrm{meV}$ ). The exciton temperature at the trap center is essentially equal to the lattice temperature due to absence of heating sources at the trap center. The estimate shows that for the excitation $P_{\mathrm{ex}}=1000 \mu \mathrm{W}$ and temperature $T_{\mathrm{b}}=1.4 \mathrm{~K}$ (see the experimental data in Fig. 2) the exciton degeneracy at the trap center is $N_{E=0} \simeq$ 8 . The theoretical modeling presented below confirms this estimate.

Note that the exciton trapping by laser light is based on a different physical principle compared to the atom trapping by laser light. However, the two techniques lead to conceptually similar optical trapping of quantum gases - of excitons or atoms, respectively.

A parabolic energy trap is apparent in the interior of the excitation ring [Figs. 1(b) and 2(c)]. The decrease in the indirect exciton PL at the location of the excitation ring [Fig. 2(a)] is because the high-energy photogenerated excitons heat the exciton gas; this heating reduces the fraction of optically active excitons, which have low energies, $E \leq$ $E_{\gamma}=E_{\mathrm{g}}^{2} \varepsilon_{\mathrm{b}} /\left(2 m c^{2}\right)$ where $E_{\mathrm{g}}$ is the energy gap and $c$ is the speed of light [27]. As they drift and diffuse away from the excitation area, the excitons thermalize to the lattice temperature $T_{\mathrm{b}}$ and become optically active, leading to the

moderately enhanced PL intensity directly external to the excitation ring. The strong enhancement of the PL at the excitation ring center, Fig. 2(a), is due to (1) the excitons' thermalization to the lattice temperature and (2) the accumulation of large numbers of excitons driven by dipole repulsion away from the higher density region towards the ring center.

A particular feature of the trap is that it is formed by the indirect excitons themselves: The trap potential is given in the mean-field approximation by $U_{\text {trap }}=\delta E=u_{0} n_{2 \mathrm{D}}=$ $4 \pi e^{2} n_{2 \mathrm{D}} d / \varepsilon_{\mathrm{b}}$, where $u_{0}$ is a positive scattering amplitude. Note that the trap confining potential is determined by the radial exciton density distribution and is essentially independent of other characteristics of indirect excitons such as their temperature, etc.

In order to model accumulation and thermalization of the particles in the laser-induced trap, we solve numerically three coupled nonlinear equations which describe the transport [25], relaxation and PL dynamics [26] of indirect excitons, respectively:

$$
\begin{aligned}
\frac{\partial n_{2 \mathrm{D}}}{\partial t}= & \nabla\left[D_{\mathrm{x}}^{(2 \mathrm{D})} \nabla n_{2 \mathrm{D}}+\mu_{\mathrm{x}}^{(2 \mathrm{D})} n_{2 \mathrm{D}} \nabla\left(u_{0} n_{2 \mathrm{D}}+U_{\mathrm{QW}}\right)\right] \\
& -\frac{n_{2 \mathrm{D}}}{\tau_{\mathrm{opt}}}+\Lambda
\end{aligned}
$$

$$
\begin{gathered}
\frac{\partial}{\partial t} T=\left(\frac{\partial T}{\partial t}\right)_{n_{2 \mathrm{D}}}+S_{\mathrm{pump}}+S_{\mathrm{opt}}+S_{\mathrm{d}}, \quad \text { where }\left(\frac{\partial T}{\partial t}\right)_{n_{2 \mathrm{D}}}= \\
-\frac{2 \pi}{\tau_{\mathrm{sc}}}\left(\frac{T^{2}}{T_{\mathrm{dB}}}\right)\left(1-e^{-T_{\mathrm{dB}} / T}\right) \int_{1}^{\infty} d \varepsilon \varepsilon \sqrt{\frac{\varepsilon}{\varepsilon-1}} \frac{\left|F_{z}(a \sqrt{\varepsilon(\varepsilon-1)})\right|^{2}}{\left(e^{\left.\varepsilon E_{0} / k_{\mathrm{B}} T_{\mathrm{b}}-1\right)}\right.} \\
\times \frac{e^{\varepsilon E_{0} / k_{\mathrm{B}} T_{\mathrm{b}}}-e^{\varepsilon E_{0} / k_{\mathrm{B}} T}}{\left(e^{\varepsilon E_{0} / k_{\mathrm{B}} T}+e^{-T_{\mathrm{dB}} / T}-1\right)} \\
\frac{1}{\tau_{\mathrm{opt}}}=\frac{1}{2 \tau_{\mathrm{R}}}\left(\frac{E_{\gamma}}{k_{\mathrm{B}} T_{\mathrm{dB}}}\right) \int_{0}^{1} \frac{1+z^{2}}{\left[\left(e^{E_{\gamma} / k_{\mathrm{B}} T}\right) /\left(1-e^{-T_{\mathrm{dB}} / T}\right)\right] e^{-z^{2} E_{\gamma} / k_{\mathrm{B}} T}-1} d z
\end{gathered}
$$

In the quantum diffusion Eq. (1), $D_{\mathrm{x}}^{(2 \mathrm{D})}, \mu_{\mathrm{x}}^{(2 \mathrm{D})}$, and $\Lambda$ are the diffusion coefficient, mobility, and generation rate of indirect excitons, respectively, $U_{\mathrm{QW}}=U_{\text {rand }}\left(\mathbf{r}_{\|}\right)$is a random potential due to the QW thickness and alloy fluctuations. The generalized Einstein relationship [25], $\mu_{\mathrm{x}}^{(2 \mathrm{D})}=D_{\mathrm{x}}^{(2 \mathrm{D})}\left[\left(e^{T_{\mathrm{dB}} / T}-1\right) /\left(k_{\mathrm{B}} T_{\mathrm{dB}}\right)\right]$, yields the classical limit $\mu_{\mathrm{x}}^{(2 \mathrm{D})}=D_{\mathrm{x}}^{(2 \mathrm{D})} /\left(k_{\mathrm{B}} T\right)$ for $T \gg T_{\mathrm{dB}}$ and strongly enhances the nonlinearity of the diffusion equation (1) for $T \lesssim T_{\mathrm{dB}}$. In Eq. (2) for effective temperature $T$ of indirect excitons, the first term on the right hand side describes the LA-phonon assisted thermalization of the particles, with $\tau_{\mathrm{sc}}=\left(\pi^{2} \hbar^{4} \rho\right) /\left(D_{\mathrm{dp}}^{2} m^{3} v_{\mathrm{s}}\right)$ the characteristic scattering time, $E_{0}=2 m v_{\mathrm{s}}^{2}, v_{\mathrm{s}}$ the longitudinal sound velocity, $\rho$ the crystal density, and $D_{\mathrm{dp}}$ the deformation potential of exciton-LA-phonon interaction. The form-factor $F_{z}(a \sqrt{\varepsilon(\varepsilon-1)})$ refers to a $\mathrm{QW}$ confinement potential, where $a=\left(d_{\mathrm{QW}} m v_{\mathrm{s}}\right) / \hbar, d_{\mathrm{QW}}$ is the $\mathrm{QW}$ thickness, and $\varepsilon=E / E_{0}$. The terms $S_{\text {pump }}, S_{\text {opt }}$, and $S_{\mathrm{d}}$ on the right hand side of Eq. (2) describe heating of indirect excitons by the laser field, recombination heating or cooling of the particles, and heating of excitons due to conversion of the mean-field energy into the internal one, respectively [28].
The optical lifetime $\tau_{\text {opt }}$ of indirect excitons is given by Eq. (3) with $\tau_{\mathrm{R}}$ the intrinsic radiative lifetime of the particles.

The quantum-statistical corrections, which considerably enhance the nonlinear effects, are explicitly included in Eqs. (1)-(3) through $T_{\mathrm{dB}} \propto \hbar^{2}$. The values of the diffusion coefficient $D_{\mathrm{x}}^{(2 \mathrm{D})}$ and the amplitude of the disorder potential $U_{\text {rand }}$ in the CQW structure were evaluated in the earlier study [28] of the inner PL ring from indirect excitons generated by a point-focused laser beam. The numerical calculations with Eqs. (1)-(3) excellently match the experimental results [Figs. 2(b) and 2(d)].

The increase of the exciton gas temperature due to heating by photogenerated excitons at the excitation ring is evident in Fig. 3(a). A minor heating due to the exciton potential energy gradient $\left[S_{\mathrm{d}}\right.$ term in Eq. (2)] can also be seen outside the excitation ring. The exciton transport towards the trap center due to drift and diffusion is illustrated in Fig. 3(c). Finally, the theoretical calculations confirm that in our experiments, which deal with the cryostat temperature $T_{\mathrm{b}}=1.4 \mathrm{~K}$, high nonclassical occupation numbers, $N_{E=0} \simeq 8$, build up at the trap center [Fig. 3(b)]. 


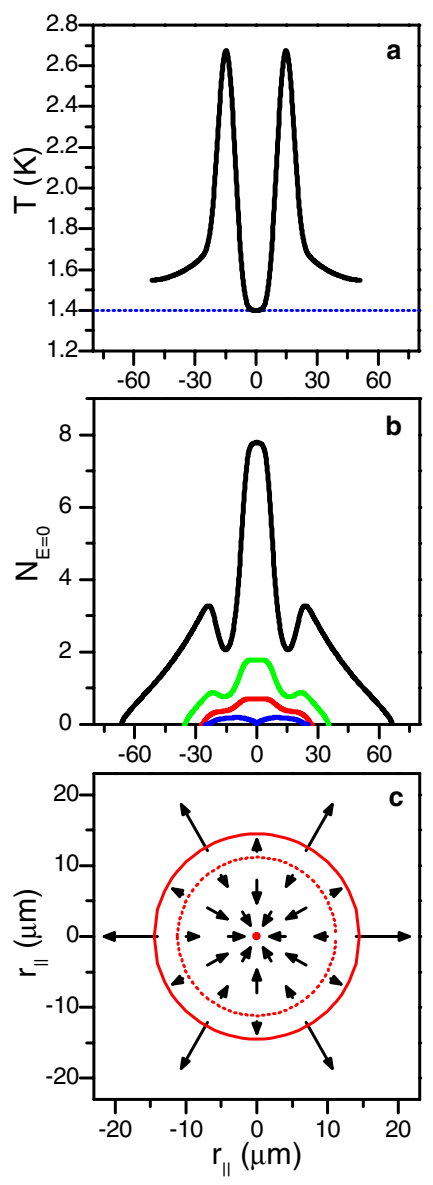

FIG. 3 (color online). The calculated parameters of excitons in the laser-induced traps. (a) The radial dependence of the exciton temperature $T$ for optical excitation power of $1000 \mu \mathrm{W}$ (solid black line) and the lattice temperature $T_{\mathrm{b}}=1.4 \mathrm{~K}$ [dotted line (blue online)]. (b) The occupation number $N_{E=0}$ for our samples against radius $r_{\|}$for the same four optical excitation powers as in Fig. 2. (c) Vector plot showing the calculated exciton velocities $v$ for excitation power of $250 \mu \mathrm{W}$. The length of the outermost vectors is $1.3 \times 10^{6} \mathrm{~cm} / \mathrm{s}$. The dotted line (red online) shows the maximum calculated exciton concentration and the solid line (red online) shows the maximum of incident laser power used in calculation.

In summary, in this Letter we propose and demonstrate a method to trap cold exciton gases with laser light. The laser-induced exciton trapping makes it possible to control the trap in situ by varying the laser intensity in space and time. The excitons at the trap center are cold since they are far from the hot laser excitation ring. We report on the trapping of a cold gas of excitons in a laser-induced trap and on the formation of a highly degenerate Bose gas of excitons in the trap.

We thank L. V. Keldysh, L. J. Sham, and D. E. Smith for valuable discussions. This work was supported by ARO Grant No. W911NF-05-1-0527 and by EU RTN Project No. HPRN-2002-00298.
[1] S. Chu, Rev. Mod. Phys. 70, 685 (1998).

[2] C. N. Cohen-Tannoudji, Rev. Mod. Phys. 70, 707 (1998).

[3] W. D. Phillips, Rev. Mod. Phys. 70, 721 (1998).

[4] A. Ashkin, IEEE J. Sel. Top. Quantum Electron. 6, 841 (2000).

[5] M. J. Lang and S. M. Block, Am. J. Phys. 71, 201 (2003).

[6] T. W. Hänsch and A. L. Schawlow, Opt. Commun. 13, 68 (1975).

[7] M.H. Anderson, J.R. Ensher, M.R. Matthews, C.E. Wieman, and E. A. Cornell, Science 269, 198 (1995).

[8] C. C. Bradley, C. A. Sackett, J. J. Tollett, and R. G. Hulet, Phys. Rev. Lett. 75, 1687 (1995).

[9] K. B. Davis, M.-O. Mewes, M.R. Andrews, N. J. van Druten, D. S. Durfee, D. M. Kurn, and W. Ketterle, Phys. Rev. Lett. 75, 3969 (1995).

[10] M. Greiner, O. Mandel, T. Esslinger, T. W. Hansch, and E. Bloch, Nature (London) 415, 39 (2002).

[11] L. V. Keldysh and A. N. Kozlov, Zh. Eksp. Teor. Fiz. 54, 978 (1968) [Sov. Phys. JETP 27, 521 (1968)].

[12] E. A. Cornell and C.E. Wieman, Rev. Mod. Phys. 74, 875 (2002).

[13] W. Ketterle, Rev. Mod. Phys. 74, 1131 (2002).

[14] L. V. Butov, J. Phys. Condens. Matter 16, R1577 (2004).

[15] J.P. Wolfe, W. L. Hansen, E. E. Haller, R. S. Markiewicz, C. Kittel, and C.D. Jeffries, Phys. Rev. Lett. 34, 1292 (1975).

[16] D. P. Trauernicht, A. Mysyrowicz, and J.P. Wolfe, Phys. Rev. B 28, 3590 (1983).

[17] K. Kash, J.M. Worlock, M.D. Sturge, P. Grabbe, J.P. Harbison, A. Scherer, and P. S. D. Lin, Appl. Phys. Lett. 53, 782 (1988).

[18] K. Brunner, U. Bockelmann, G. Abstreiter, M. Walther, G. Böhm, G. Tränkle, and G. Weimann, Phys. Rev. Lett. 69, 3216 (1992).

[19] P. C. M. Christianen, F. Piazza, J. G. S. Lok, J.C. Maan, and W. van der Vleuten, Physica (Amsterdam) 249B, 624 (1998).

[20] S. Zimmermann, A. O. Govorov, W. Hansen, J.P. Kotthaus, M. Bichler, and W. Wegscheider, Phys. Rev. B 56, 13414 (1997).

[21] T. Huber, A. Zrenner, W. Wegscheider, and M. Bichler, Phys. Status Solidi (a) 166, R5 (1998).

[22] A. T. Hammack, N. A. Gippius, Sen Yang, G. O. Andreev, L. V. Butov, M. Hanson, and A. C. Gossard, J. Appl. Phys. 99, 066104 (2006).

[23] D. Yoshioka and A. H. MacDonald, J. Phys. Soc. Jpn. 59, 4211 (1990).

[24] X. Zhu, P. B. Littlewood, M. Hybertsen, and T. M. Rice, Phys. Rev. Lett. 74, 1633 (1995).

[25] A. L. Ivanov, Europhys. Lett. 59, 586 (2002).

[26] A. L. Ivanov, P. B. Littlewood, and H. Haug, Phys. Rev. B 59, 5032 (1999).

[27] J. Feldmann, G. Peter, E. O. Gobel, P. Dawson, K. Moore, C. Foxon, and R. J. Elliott, Phys. Rev. Lett. 59, 2337 (1987).

[28] A. L. Ivanov, L. E. Smallwood, A. T. Hammack, Sen Yang, L. V. Butov, and A. C. Gossard, Europhys. Lett. 73, 920 (2006). 